\title{
Calculating "g" from Acoustic Doppler Data
}

Sebastián M. Torres, The University of Oklahoma, Norman, OK

Wilson J. González-Espada, Arkansas Tech University, Russellville, AR

$\mathbf{T}$ raditionally, the Doppler effect for sound is introduced in high school and college physics courses. Students calculate the perceived frequency for several scenarios relating a stationary or moving observer and a stationary or moving sound source. These calculations assume a constant velocity of the observer and/or source. Although seldom discussed in this context, generalization of the Doppler effect for accelerated sound sources is relatively straightforward and can be used as an enriching tool in the classroom. The purpose of this paper is to describe a simple experiment to determine the acceleration of gravity $(g)$ from an acoustic source in free fall.

The Doppler effect is usually applied to scenarios where the relative velocity between a sound-emitting object and an observer is nonzero or when the perceived and real sound frequencies are known and a specific speed is unknown. ${ }^{1}$ Sometimes a combined equation can be used in which the user changes positive and negative signs depending on the relative motions of the sound-emitting object and the observer. That is,

$$
f^{*}=f \frac{v \pm v_{0}}{v \pm v_{\mathrm{s}}}
$$

where $f^{*}$ is the Doppler-shifted frequency, $f$ is the frequency of the acoustic source, $v$ is the speed of sound (in air at $20^{\circ} \mathrm{C}, 343.6 \mathrm{~m} \cdot \mathrm{s}^{-1}$ ), and $v_{0}$ and $v_{\mathrm{s}}$ are the speeds of the observer and the source, respectively.

Although it is commonplace to find discussions about the Doppler effect with sound sources in circu- lar motion, ${ }^{2,3}$ most textbooks do not discuss sound sources or observers increasing or decreasing their speed at a constant rate. Interestingly, one-dimensional kinematics with constant acceleration is another common topic discussed in introductory physics courses, and generalization of the Doppler effect for accelerated sound sources or observers is relatively straightforward. In this article we will examine the case of a stationary observer $\left(v_{0}=0\right)$ and an accelerated sound source $\left(v_{s}=a t\right)$. In this case, Eq. (1) can be modified as

$$
f^{*}(t)=f \frac{v}{v \pm a t}
$$

where $a$ is the acceleration of the sound source and $f^{*}$ is now a function of time $t$. In theory, the Doppler-shifted frequency of free-falling sound sources could be used as a means to determine the acceleration of gravity, with $a=g$ in the previous equation.

\section{Previous Attempt}

A previous attempt to measure the acceleration of gravity using experimental Doppler data was reported by one of the authors in $2002 .{ }^{4}$ Given that the final product was supposed to be used in a high school physics laboratory, the experimental design was very simple. A buzzer was located close to a microphone placed about two meters from the floor. The microphone was connected to a computer with a standard sound card running a sound analyzing program. Due to the analysis limitations of the software, 


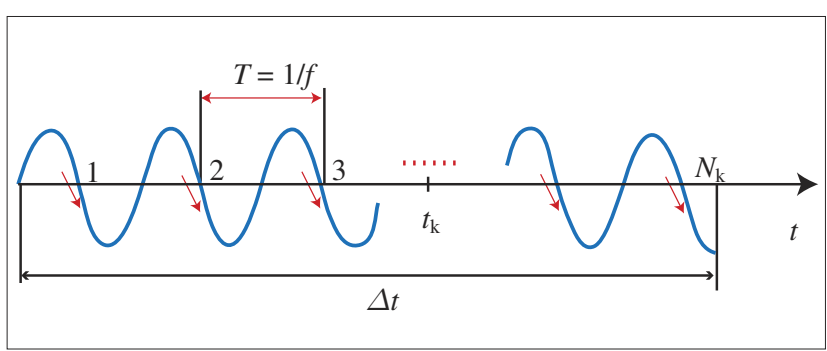

Fig. 1. Depiction of instantaneous frequency estimation using zero crossings of the signal. Each zero crossing is indicated with an arrow and numbered from 1 to $N_{k}$. The number of zero crossings in the interval $\Delta t$ is used to estimate the instantaneous frequency of the signal at time $\boldsymbol{t}_{\boldsymbol{k}}$.

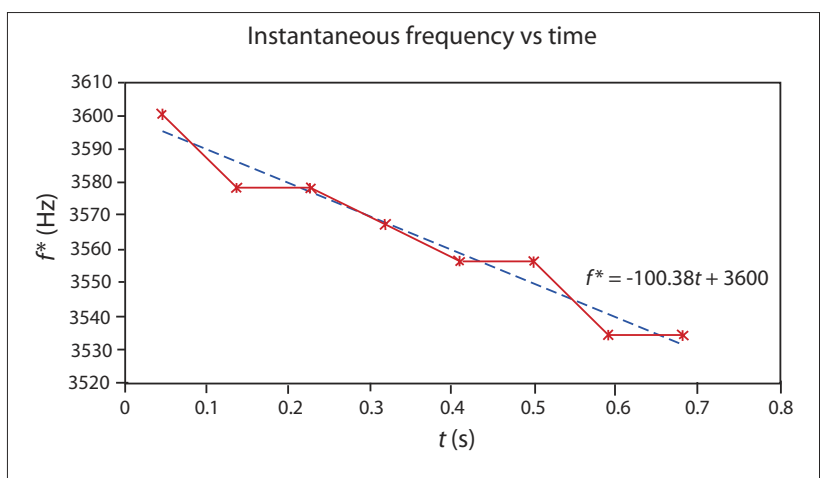

Fig. 2. Instantaneous Doppler-shifted frequency vs time obtained from a model simulating a free-falling 3600 $\mathrm{Hz}$ buzzer and analyzed with a spreadsheet program (Microsoft Excel). The solid line corresponds to $f^{*}$ measured from the data and the dashed line to the computed linear trend. The equation of the linear trend from which to get the slope of the curve is readily obtained using the spreadsheet built-in functions.

the changes in wave frequency were determined by manually counting periods of the measured wave on several printouts encompassing the entire falling time (each printout was used to obtain the "instantaneous" frequency at a given time interval). In that case, the determination of the acceleration of gravity resulted in an average value of $6.4 \mathrm{~m} \cdot \mathrm{s}^{-2}$, about $35 \%$ below $9.8 \mathrm{~m} \cdot \mathrm{s}^{-2}$, the value of $g$ commonly used. Proposed sources of error included the time-frequency uncertainty principle, ${ }^{5}$ reverberation and interference in the laboratory, possible buzzer rotation as it fell, and the limitations of the equipment to measure very small frequency changes over a short period of time. Not surprisingly, others have also described the Doppler effect for accelerated sound sources as "difficult to examine experimentally in the time domain." 6

\section{New Experiment}

In this paper, we propose a more promising approach for the experimental determination of $g$ using acoustic Doppler data. The fundamental problem consists of measuring the time-varying Dopplershifted frequency of the free-falling sound source. Accordingly, we must first establish a suitable definition of instantaneous frequency. In general, defining the instantaneous frequency for nonstationary signals is controversial and dependent on the application. As a result, this issue has received significant attention in the field of digital signal processing and complex techniques exist. ${ }^{7}$ Nevertheless, we chose a simple analysis approach that is suitable for the high school classroom. For our application, the instantaneous Doppler-shifted frequency $f^{*}$ is defined as the average frequency during a small interval of time $\Delta t$. Thus,

$$
f^{*}\left(t_{k}\right) \approx \frac{N_{k}}{\Delta t}
$$

where $t_{k}$ is the center of the $k$-th time interval, and $N_{k}$ is the number of zero crossings of the signal going from a positive to a negative value (Fig. 1). Note that Eq. (3) assumes that the signal is locally stationary within $\Delta t$. Therefore, there is a trade-off in choosing $\Delta t$. This interval must be long enough to minimize the errors in Eq. (3) by encompassing several cycles of the signal, but at the same time short enough to accommodate the assumption of local stationarity. For our analysis, we found that good results can be obtained by dividing the total recorded signal (about $0.65 \mathrm{~s}$ ) into seven to 10 equal parts.

Because the maximum Doppler shift is much smaller than the rest frequency of the buzzer, Eq. (2) can be estimated using a first-order approximation (taking the first two terms of the Maclaurin series): ${ }^{8}$

$$
f^{*}(t) \approx f-\frac{g f}{v} t
$$

Therefore, $g$ can be obtained from the slope of $f^{*}(t)$ as

$$
g=-\operatorname{slope}\left(\frac{v}{f}\right) .
$$

In practice, a simple way to do this is to graph $f^{*}$ versus time and then obtain a linear trend (linear fit) from which to get the slope. 


\section{Theoretical Results}

The results in Fig. 2 were obtained with simulated data. The model for the signal $S(t)$ received by the microphone consists of a sinusoidal wave with amplitude that decreases quadratically as the sound source falls and instantaneous frequency given by Eq. (2). That is,

$$
S(t)=A(t) \sin \varphi(t) .
$$

In this equation, $A$ is inversely proportional to the square of the distance $h$ between the microphone and the free-falling buzzer; i.e., $A=1 / h^{2}$ with $h=$ $g t^{2} / 2$. On the other hand, the instantaneous frequency of $S(t)$ is proportional to the derivative of the argument of the sine function: ${ }^{9}$

$$
f^{*}(t)=\frac{1}{2 \pi} \frac{d \varphi(t)}{d t} .
$$

Equating Eqs. (7) and (2) one can integrate both sides to obtain $\varphi$ in Eq. (6). Finally, substituting the expressions for $A$ and $\varphi$, the model for the signal received by the microphone is

$$
S(t)=\frac{4}{g^{2} t^{4}} \sin \left[2 \pi \frac{f v}{g} \ln \left(1+\frac{g t}{v}\right)\right] .
$$

Using this model, a speed of sound of $343.6 \mathrm{~m} \mathrm{~s}^{-1}$, a buzzer frequency of $3600 \mathrm{~Hz}$, and the procedure outlined above, the slope of best-fit line from the instantaneous frequency and time graph is estimated as $-100.38 \mathrm{~s}^{-2}$ (Fig. 2), resulting in a value of $g$ of $9.58 \mathrm{~m} \cdot \mathrm{s}^{-2}$. This amount is about $2.2 \%$ off the commonly used value of $9.8 \mathrm{~m} \cdot \mathrm{s}^{-2}$.

\section{Experimental Results}

To confirm whether the theoretical model matches real data-collection conditions, the authors used an experimental setup similar to González-Espada and Robertson. ${ }^{4}$ Sound acquisition was done with the standard Microsoft Windows "Sound Recorder" utility. For proper sound recording, the sound card was set for one-channel (mono) recording using 8 bits per sample point and a sampling frequency $\left(f_{\mathrm{s}}\right)$ of at least twice the frequency of the buzzer $(f)$. In our case, a sampling frequency of $11025 \mathrm{~Hz}$ was used.

The experiment was divided into two parts. First, the rest frequency of the buzzer was measured and compared with previous measurements. Second, the

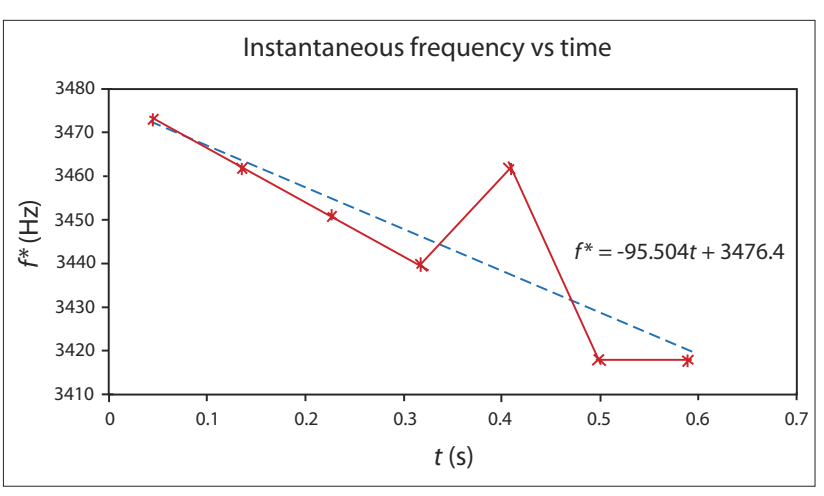

Fig. 3. Instantaneous Doppler-shifted frequency vs time obtained from experimental data analyzed with a spreadsheet program. The solid line (and its associated equation) corresponds to $f^{*}$ measured from the data and the dashed line to the computed linear trend.

buzzer was dropped several times to collect sound data for the duration of the falls (only one result will be shown here). Data sets were exported to a spreadsheet (e.g., Microsoft Excel) for their analysis. Because "Sound Recorder" cannot save the recorded data as text, we used a freeware tool called SoX - Sound Exchange (available from http://sox.sourceforge.net) to convert the data. The rest frequency of the buzzer was measured at $3498.23 \mathrm{~Hz}$, about $100 \mathrm{~Hz}$ lower than measurements performed in 2002. Using the graphs of instantaneous frequency as a function of time, a slope of $-95.504 \mathrm{~s}^{-2}$ was obtained for the experiment shown in Fig. 3. From Eq. (5) a value for the acceleration of gravity was calculated as $9.42 \mathrm{~m} \mathrm{~s}^{-2}$, a vast improvement over previous attempts to determine $g$ using the Doppler effect.

Interestingly, the average frequency for the time interval around $0.4 \mathrm{~s}$ appears to diverge from the trend suggested by the rest of the data points. The authors do not have an explanation for the apparent difference, although it is possible that buzzer rotation might have affected our measurements.

The reader interested in replicating this experiment should ensure that the microphone points downward. The authors spent several hours analyzing echo frequencies (without Doppler shift) because the microphone was inadvertently placed at an angle. Interestingly, higher sampling frequencies were also used $(16,000 \mathrm{~Hz})$, but a Doppler shift could not be detected experimentally. It is theorized that ambient interference and room echoes caused more contamination of the buzzer signal at this sampling frequency. 


\section{Conclusions and Perspectives}

A number of interesting questions remain and can easily be converted into student research projects. For example, the influence of the falling buzzer's rotation in the quality of sound data collected by the microphone is unknown. Further, it might be possible to obtain better results with a higher-frequency buzzer, a more expensive microphone, spectrogram software, or by conducting the experiment outdoors to avoid reverberance. Finally, to better match the proposed signal model in future experiments, a colleague proposed using a small speaker connected to a pure sine generator instead of a buzzer that might contain higher-order harmonics.

We demonstrated that it is possible to use a standard PC microphone and simple processing techniques to measure the acceleration of gravity by detecting small frequency changes (from $3500 \mathrm{~Hz}$ to $3450 \mathrm{~Hz}$ ) over a period of less than a second. The experimental corroboration of the results obtained with simulated data is evidence that this process can be replicated without expensive equipment, an important consideration for many high school physics laboratories.

\section{Acknowledgment}

This paper was prepared by Sebastián Torres and Wilson González with partial funding provided by NOAA/Office of Oceanic and Atmospheric Research under NOAA-University of Oklahoma Cooperative Agreement \#NA17RJ1227, U.S. Department of Commerce. The statements, findings, conclusions, and recommendations are those of the authors and do not necessarily reflect the views of NOAA or the U.S. Department of Commerce.

\section{References}

1. D. Halliday and R. Resnick, Fundamentals of Physics, 3rd ed. extended (Wiley, New York, 1988).

2. Marcelo M.F. Saba and Rafael Antônio da S. Rosa, "The Doppler effect of a sound source moving in a circle," Phys. Teach. 41, 89-91 (Feb. 2003).

3. Thomas D. Rossing, "The Doppler effect and racing cars," Phys. Teach. 26, 423 (Oct. 1988).

4. Wilson J. González-Espada and Jeff Robertson, "The Doppler effect for a constantly accelerating sound source receding from a stationary observer: Determining the acceleration of gravity based on acoustic data," paper presented at the Annual Meeting of the Arkansas
Academy of Science (April 2002).

5. K. Grochenig, Foundations of Time-frequency Analysis (Birkhauser, Boston, 2001).

6. T.J. Bensky and S.E. Frey, "Computer sound card assisted measurements of the acoustic Doppler effect for accelerated and unaccelerated sound sources," Am. J. Phys. 69, 1231 (Dec. 2001).

7. Boualem Boashash, "Estimating and interpreting the instantaneous frequency of a signal - Part 1: Fundamentals," Proc. IEEE 80, 520 (April 1992).

8. An equivalent approach that might be more effective for algebra-based physics students is to use an approximation of the Doppler formula for low speeds ${ }^{12}$ : $\Delta f / f \approx v_{\mathrm{R}} / v$, where $\Delta f$ is the Doppler shift and $v_{\mathrm{R}}$ is the relative velocity between the observer and the source. From this equation and using the relative velocity corresponding to a stationary observer and an accelerated source $\left(v_{\mathrm{R}}=g t\right)$, the Doppler shift can be approximated as $\Delta f=f-f^{*} \approx f v_{\mathrm{R}} / v=f g t / v$. This equation is the same as Eq. (4) and can be derived without the use of calculus.

9. Daniel Hoyt, "A different viewpoint on Doppler-effect calculations," Phys. Teach. 40, 16 (Jan. 2002).

PACS codes: 01.40.ek, 01.50.ht, 01.50.Pa, 06.30.Ft, 06.30.Gv, 43.90.+v

\section{Sebastián M. Torres, research scientist, Cooperative Institute for Mesoscale Meteorological Studies, The University of Oklahoma and NOAA/OAR National Severe Storms Laboratory, adjunct assistant professor, School of Electrical and Computer Engineering.}

The University of Oklahoma, 1313 Halley Circle, Norman, OK 73069; Sebastian.Torres@noaa.gov

Wilson J. González-Espada, assistant professor of physical science, Department of Physical Science, Arkansas Tech University.

Arkansas Tech University, 1701 North Boulder Ave., Russellville, AR 72801; wilson.gonzalezespad@atu.edu 